\title{
Emergence of the Reactivity Continuum of Organic Matter from Kinetics of a Multitude of Individual Molecular Constituents
}

\author{
Alina Mostovaya, ${ }^{* \dagger}{ }^{\dagger}$ Jeffrey A. Hawkes, ${ }^{\dagger}$ Birgit Koehler, ${ }^{\dagger}$ Thorsten Dittmar, $^{\S}$ and Lars J. Tranvik ${ }^{\dagger}$ \\ ${ }^{\dagger}$ Department of Ecology and Genetics/Limnology, Evolutionary Biology Centre, Uppsala University, Norbyvägen 18 D, 75236 \\ Uppsala, Sweden \\ ${ }^{\ddagger}$ Department of Chemistry-BMC, Analytical Chemistry, Uppsala University, Husargatan 3, 75124 Uppsala, Sweden \\ ${ }^{\S}$ Research Group for Marine Geochemistry (ICBM-MPI Bridging Group), Institute for Chemistry and Biology of the Marine \\ Environment, University of Oldenburg, Carl-von-Ossietzky-Strasse 9-11, 26129 Oldenburg, Germany
}

\section{Supporting Information}

ABSTRACT: The reactivity continuum (RC) model is a powerful statistical approach for describing the apparent kinetics of bulk organic matter (OM) decomposition. Here, we used ultrahigh resolution mass spectrometry data to evaluate the main premise of the RC model, namely that there is a continuous spectrum of reactivity within bulk OM, where each individual reactive type undergoes exponential decay. We performed a 120 day OM decomposition experiment on lake water, with an untreated control and a treatment preexposed to UV light, and described the loss of bulk dissolved organic carbon with RC modeling. The behavior of individual molecular formulas was described by fitting the single exponential model to the change in peak intensities over time. The range of the empirically derived apparent exponential decay coefficients $\left(k_{\text {exp }}\right)$ was indeed continuous. The character of the corresponding distribution, however, differed from the conceptual expectations, due to the effects of intrinsic averaging, overlaps in formula-specific loss and formation rates, and the limitation of the RC model to include apparently accumulating compounds in the analysis. Despite these limitations, both the RC model-simulated and empirical (mass spectrometry-derived) distributions of $k_{\text {exp }}$ captured the effects of preexposure to UV light. Overall, we present experimental evidence that the reactivity continuum within bulk OM emerges from a range of reactivity of numerous individual components. This constitutes direct empirical support for the major assumption behind the RC model of the natural OM decomposition.

\section{INTRODUCTION}

Organic matter (OM) is a major component of the carbon cycle. The complex nature of OM was suggested in multiple studies demonstrating that different fractions of OM decompose at differing rates. ${ }^{1-4}$ With the increasing understanding of OM complexity, OM was conceptualized as a continuous spectrum of constituents with differing reactivity, ${ }^{5-7}$ long before it was possible to identify the broad range of OM components. Recent advances in analytical chemistry, and particularly high-resolution mass spectrometry, have demonstrated that natural $\mathrm{OM}$ may consist of $10^{4}-10^{6}$ compounds, belonging to different chemical classes and groups, ${ }^{8-11}$ yet the emerging detailed insights into OM composition have not been explicitly connected to the concept of the reactivity continuum.

The reactivity continuum (RC) model approach is gaining popularity as a tool for realistic description of decomposition in a variety of environments, including marine sediments, ${ }^{6,7,12}$ soil, $^{13,14}$ aquatic dissolved OM, ${ }^{15-17}$ and litter. ${ }^{18,19}$ The RC model was often shown to perform better than single- and multiexponential models, based on mathematical indicators assessing accuracy and parsimony, ${ }^{14,16,18,20}$ as well as the predictive power of the model. ${ }^{15}$ A study analyzing the Longterm Intersite Decomposition Experiment (LIDET data) with $\mathrm{RC}$ modeling showed that the obtained RC model parameters were suitable to predict independent literature-derived decomposition time series for the majority of litter-biome combinations. ${ }^{18}$ Because the RC model can successfully track the effects of external regulators of decomposition, ${ }^{18,20}$ it has been proposed to improve the mechanistic understanding of decomposition at large spatial and temporal scales. ${ }^{18}$ Accordingly, Aumont et al. (2017) used the RC model in a global ocean biogeochemical model to account for variable reactivity of particulate organic carbon, and got a close match to empirical observations. $^{12}$

The RC model assumes that decomposition of bulk OM can be described as an integral of single-pool exponential decay functions weighted by an initial probability distribution of

Received: June 5, 2017

Revised: August 12, 2017

Accepted: September 1, 2017

Published: September 15, 2017 
reactivity. When the chosen functional shape for the initial probability distribution of reactivity allows a simple analytical solution (e.g., gamma distribution) the RC model becomes a parameter-parsimonious and computationally simple approach to analyze decomposition data. The main gamma RC model assumptions ${ }^{6}$ are as follows:

(1) Each individual reactive type decays over time following a single-pool exponential model;

(2) The individual reactive types form a continuous spectrum of initial reactivity toward decomposition, that can be described by a probability distribution; and

(3) The gamma distribution is a flexible and suitable function to describe the initial probability distribution of reactivity.

Since the high-resolution analytical techniques that could provide detailed information on the behavior of $\mathrm{OM}$ constituents became available only recently, these RC model assumptions so far remain empirically untested. With the recent development of ultrahigh-resolution electrospray ionization Fourier transform ion cyclotron resonance mass spectrometry (FT-ICR-MS), which is currently one of the most powerful analytical techniques for molecular characterization of natural $\mathrm{OM}^{21-23}$ thousands of molecular formulas can now be identified in environmental samples. Further, the signal intensity of each molecular mass is essentially linearly proportional to the concentration of the corresponding isomeric mixture of compounds, provided that the sample matrix is similar. ${ }^{24}$ This allows interpreting the changes in signal intensity over time in a quantitative way. Therefore, using FTICR-MS, we were able to experimentally investigate one of the main assumptions of the $\mathrm{RC}$ model that individual reactive types form a continuum of decay rates within bulk OM.

Taking decomposition of lake water dissolved organic matter (DOM) as a case, we performed a 120 days long decomposition experiment. We incubated untreated lake water as well as lake water subjected to UV exposure to stimulate DOM decomposition. Decomposition of bulk DOM was analyzed with the gamma RC model, obtaining the respective probability distribution of initial decay coefficients $k .^{20}$ In this study, we fitted the single-pool exponential model to each time series of intensities related to individual molecular formulas, and extracted the corresponding apparent exponential decay coefficients $k_{\text {exp. }}$. We compare the range of RC modelsimulated and empirically derived initial decay coefficients, and discuss our results with respect to the assumptions of the RC model. Our results support the existence of a reactivity continuum within DOM and provide an argument in favor of continuous models of OM decay. In the context of potential application of our approach, further discussion reveals the advantages and limitations of using ultrahigh resolution mass spectrometry for evaluating the kinetics of OM decay.

\section{METHODS}

2.1. Experimental Setup. Waters from Lakes Ramsjön $\left(59^{\circ} 50^{\prime} \mathrm{N}, 17^{\circ} 13^{\prime} \mathrm{E}\right)$ and Övre Långsjön $\left(59^{\circ} 52^{\prime} \mathrm{N}, 18^{\circ} 01^{\prime} \mathrm{E}\right)$, located in east-central Sweden, were sampled in October 2013. Water samples were taken from the surface (0-0.5 m depth), $0.2 \mu \mathrm{m}$ filtered (Supor 200, Pall Corporation), and kept in the dark at $4{ }^{\circ} \mathrm{C}$ for 3 weeks prior to the start of decomposition experiments. These 3 weeks were required to expose portions of the filtered water to artificial UV light ( $144 \mathrm{~h}$ under a UV lamp providing an irradiance of $35 \mathrm{~W} \mathrm{~m}^{-2}$ between 300 and
$400 \mathrm{~nm}$ ), which resulted in photodegradation of about $30 \%$ of dissolved organic carbon (DOC).$^{20}$ Prior to the incubations the filtered water was inoculated with a $64 \mu \mathrm{m}$ plankton net filtered aliquot from the respective lake ( $5 \%$ of the volume) and amended with inorganic nutrients $\left(480 \mu \mathrm{g} \mathrm{N} \mathrm{L}^{-1}\right.$ as $\mathrm{KNO}_{3}$ and $100 \mu \mathrm{g} \mathrm{P} \mathrm{L}^{-1}$ as $\mathrm{Na}_{2} \mathrm{HPO}_{4}$ ). The water was incubated at $20^{\circ} \mathrm{C}$ in sealed $40 \mathrm{~mL}$ glass vials capped with PTFE-lined silicone septa. The vials were filled headspace free and submersed in water to minimize gas exchange. The incubations were conducted in the dark and lasted for 120 days. Each of the two treatments (nonmanipulated and UV-manipulated) was duplicated within each lake, which yielded 8 time series of DOC loss. In the course of decomposition experiment individual vials in each time series were sacrificed to measure DOC concentration and take subsamples for mass spectrometry analysis (Supporting Information (SI) Figure S1). The DOC concentrations were measured on a Sievers 900 TOC Analyzer (General Electric Analytical Instruments, Manchester, U.K.) at 20 and 18 time points in the nonmanipulated and UVmanipulated treatment, respectively. ${ }^{20}$ Samples for mass spectrometry analysis were taken at 12 time points: water was transferred into $2 \mathrm{~mL}$ glass vials with PTFE-lined silicone septa and stored at $4{ }^{\circ} \mathrm{C}$ prior to analysis. Before use, $40 \mathrm{~mL}$ glass vials were acid-washed $(10 \% \mathrm{HCl})$, rinsed with Milli-Q water, and precombusted for $4 \mathrm{~h}$ at $550{ }^{\circ} \mathrm{C}$. The $2 \mathrm{~mL}$ glass vials were precombusted for $4 \mathrm{~h}$ at $450{ }^{\circ} \mathrm{C}$. All plastic caps and silicone septa were presoaked in methanol for $24 \mathrm{~h}$ and repeatedly rinsed with Milli-Q water.

2.2. Mass Spectrometry Analysis. Lake water aliquot volumes were adjusted by diluting the sample with ultrapure water to a concentration of $10 \mathrm{mg} \mathrm{C} \mathrm{L}{ }^{-1}$. Further, the aliquots were mixed with methanol (HPLC-grade, Sigma-Aldrich) in a proportion of $2: 1$ (final concentration $6.7 \mathrm{mg} \mathrm{C} \mathrm{L}^{-1}$ ), refiltered (0.2 $\mu \mathrm{m}$ PTFE), and introduced into a $15-\mathrm{T}$ ultrahighresolution electrospray ionization Fourier transform ion cyclotron resonance mass spectrometer (FT-ICR-MS; Bruker Daltonics) at $360 \mu \mathrm{L} \mathrm{h}^{-1}$. Electrospray ionization (ESI) was performed in negative mode. Mass spectra were collected over 400 scans, with an ion accumulation time of $0.5 \mathrm{~s}$, and within a range of $150-2000 \mathrm{~m} / z$. Each sample was individually calibrated with an internal reference mass list generated from North Equatorial Pacific Intermediate Water (NEqPIW $)^{25}$ using the Bruker Daltonics Data Analysis software package. Only peaks with a signal-to-noise ratio greater than four were considered. Where possible, peaks were assigned a formula containing $\mathrm{C}, \mathrm{H}, \mathrm{O}, \mathrm{N}, \mathrm{S}$, and $\mathrm{P}$ based on the following conditions: $\mathrm{C} \geq \mathrm{O} ; \mathrm{O}>(2 \mathrm{P}+\mathrm{S}) ; \mathrm{N} \leq 4 ; \mathrm{S} \leq 4$ and $\mathrm{P} \leq 1$. $\mathrm{CHO}$ compounds had corresponding peaks at +1.0034 mass units due to $1 \%$ abundance of ${ }^{13} \mathrm{C}$, with intensities confirming the formula assignment and confirming the single charge of the ion. Formulas containing S, P, and $\mathrm{N}>1$ were not associated with any systematic patterns in apparent exponential coefficients $k_{\text {exp }}$, i.e., their presence did not affect the shape of empirical distribution of $k_{\exp }$ (see section 2.3). Therefore, to minimize false positives, i.e., possible incorrect assignments, we used a more conservative approach which does not consider S-, $\mathrm{P}$-, and $\mathrm{N}>1$-containing formulas. Unassigned masses were not considered, as most of them represent either isotopologues $\left(\right.$ e.g. ${ }^{13} \mathrm{C}$ ) or noise (SI Figures S2 and S3, and SI Table S1). We did not observe any significant shift or systematic pattern in the amount and total intensity of unassigned peaks in the beginning versus the end of the incubations. Also, unassigned peaks were not associated with any specific patterns in $k_{\text {exp }}$. Peaks detected 
in procedural blanks, as well as the peaks commonly identified as contaminants, and peaks with unusually and nonreproducibly high intensity in samples were removed from the data set. In addition, the peaks with mass defect in the range of $0.3-0.8$ were disregarded. The peaks present in only one of the two replicates of each of the four lake-treatment combinations (i.e., each lake with and without UV pretreatment) were disregarded as well. Examples of original mass spectra from different time points and graphical representations of formula assignment are presented in SI Figures S2 and S3, respectively. The SI Table $\mathrm{S} 1$ gives details on data reduction procedures (total intensity of assigned versus unassigned peaks, etc.).

Here, before proceeding to further analysis we checked whether the time series of total intensity followed the trends in bulk DOC concentration (SI Figure S4). One of the replicate incubations did not meet this criterion and was therefore removed from further analysis. Thereafter, to account for possible variable contamination between individual time series, data from each lake-treatment-replicate combination (seven in total) was processed separately. A detection limit was applied based on dynamic range. The dynamic range of assigned peaks was determined in each sample as a ratio between maximum and minimum assigned peak intensity. Detection limit was calculated by dividing maximum intensity in each sample by the dynamic range of the worst sample. All intensities below the detection limit were disregarded. The intensities were further normalized to the sum of signal intensities in each sample and multiplied to account for dilution, i.e., to estimate the equivalent intensity in the undiluted sample. This was done to correctly estimate the relative change in concentration over the course of the decomposition experiment. ${ }^{24,26}$ For the purpose of model fitting, only the peaks that were detected on at least 8 days of the 12-points time series were considered.

Our analysis strongly relies on the assumption of a linear relationship between intensity and concentration, which was documented previously ${ }^{24}$ in a series of dilution experiments where Amazon DOM was incrementally mixed with Atlantic DOM. Around $70 \%$ of molecular formulas in the terrestrial and marine endmembers showed a significant $(p \leq 0.05)$ linear response of signal intensity according to the experimental mixing. ${ }^{24}$ For most of the remaining $30 \%$ of formulas the linear response could not be tested because their initial intensity did not differ between the Amazon and Atlantic endmembers (Thorsten Dittmar, personal communication). In our samples the major peaks were inorganic and did not decay over time (SI Figure S2). This likely provided a stable matrix, and ensured a reliable comparison of OM peaks in each time series of our samples.

To evaluate the instrument-specific noise variations we used the 26 time point measurements of an in-house DOM reference material (NEqPIW) ${ }^{25}$ (final concentration $15 \mathrm{mg} \mathrm{C} \mathrm{L}^{-1}$ ). The standard was measured in the beginning and end of every day, on 13 days in total.

2.3. Model Fitting and Statistical Analysis. The gamma $\mathrm{RC}$ model was fitted to the time series of relative DOC loss, as described in detail earlier. ${ }^{20}$ Briefly, the two model parameters, $a$ and $v$, were estimated based on the following relationship:

$$
\frac{\mathrm{DOC}_{t}}{\mathrm{DOC}_{0}}=\left(\frac{a}{a+t}\right)^{v}
$$

where $a$ is a rate parameter indicating the average lifetime of the more reactive compounds (days), and $v$ (unitless) is a shape parameter characterizing the shape of the distribution near $k=$ $0{ }^{6}$ The apparent decay coefficient $k_{t}\left(\mathrm{day}^{-1}\right)$ at a given time point is calculated as follows: ${ }^{27}$

$$
k_{t}=\frac{v}{a+t}
$$

The initial apparent first-order decay coefficient $k_{0}$ (expected value of the initial probability distribution of reactivity) is therefore defined as $v / a$.

To approach the assumption of the $\mathrm{RC}$ model from the empirical end we used mass spectrometry-derived information on signal intensities of molecular formulas and its change over time. For each formula, the relative change in signal intensity was described with a single pool exponential model: ${ }^{28}$

$$
I_{t}=I_{0} e^{-k_{\exp } t}
$$

where $k_{\exp }$ is an apparent exponential decay coefficient $\left(\right.$ day $\left.^{-1}\right)$ and $I_{t}$ simulates the change in intensity over time. Model fitting was conducted using generalized least-squares modeling to allow the errors to be correlated (R-function gnls). The goodness of model fit was evaluated using the normalized rootmean-square error (NRMSE). We used the NRMSE to filter the data sets from the time series containing a lot of noise. To establish an acceptable NRMSE we calculated the 95\% quantile of the NRMSE distribution acquired from a 26-point series of measurements of DOM standard (NEqPIW) of stable concentration $\left(15 \mathrm{mg} \mathrm{C} \mathrm{L} \mathrm{L}^{-1}\right)$. On the basis of these calculations, the threshold for NRMSE was established at $35.8 \%$, and between $89.7 \%$ and $93.6 \%$ of formulas in the actual data sets passed this criterion.

To verify that our data sets are based on nonrandom time series of intensities we compared the distribution of $k_{\exp }$ in our data sets to the distribution of $k_{\text {exp }}$ derived from an analogous but artificial data set based on random numbers. The distribution of $k_{\exp }$ in the actual data sets was more narrow and skewed toward positive values (SI Figure S5), as was also indicated by the nonparametric Mann-Whitney $U$ test $(P<$ 0.001 for all seven lake-treatment-replicate combinations), signifying that the actual data sets were indeed nonrandom.

For the bulk DOC, the probability distribution of initial reactivities was simulated using a gamma cumulative distribution function, specified by the RC model parameters $a$ and $v$. According to Boudreau and Ruddick, "the basic premise of the continuum theory is the existence of the spectrum of reactive types characterized by a distribution function $g(k, t)$ which determines the concentration of organic matter having reactivities between $k$ and $k+\mathrm{d} k$ (where $\mathrm{d} k$ is an infinite small increment of $k$ )". From the perspective of mass spectrometryderived data, the concentration share of each formula within DOM remains unknown since signal intensity is not concentration-calibrated, and therefore cannot be used to compare the concentrations of different formulas at a certain time point. It was therefore not possible to build the empirical probability distribution of initial reactivities (i.e., $k_{\text {exp }}$ ) in the same way as the RC model simulation implies. However, it was possible to fit the gamma distribution into the empirically derived range of $k_{\text {exp }}$, bearing in mind that such an approach links $k_{\exp }$ with the number of detected molecular formulas, and not with the concentration. It should also be remembered that the apparent empirical decay rates might be the net result between simultaneous loss and formation of the molecular formulas. Further, we could only use positive $k_{\exp }$ since the gamma distribution only allows for $k$ to be positive. The 

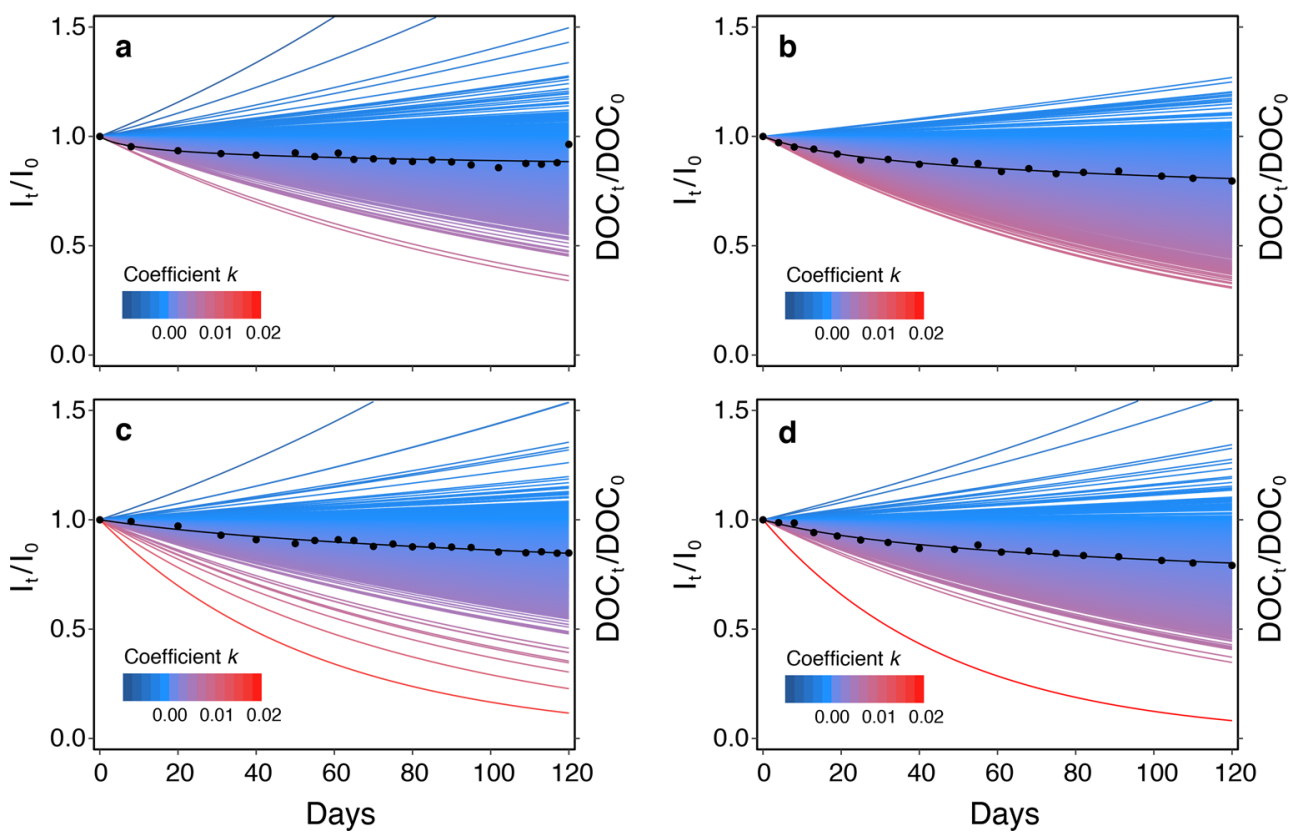

Figure 1. Relative change in intensities of individual molecular formulas over time described with single exponential model fits. Presented are data for lake Ramsjön nonmanipulated (a) and UV-manipulated (b) treatments, and lake Övre Långsjön nonmanipulated (c) and UV-manipulated (d) treatments. Only one of the duplicate treatments is shown in each case. The black line shows the gamma RC model fitted to the relative loss in bulk DOC (black dots) over time. ${ }^{20}$
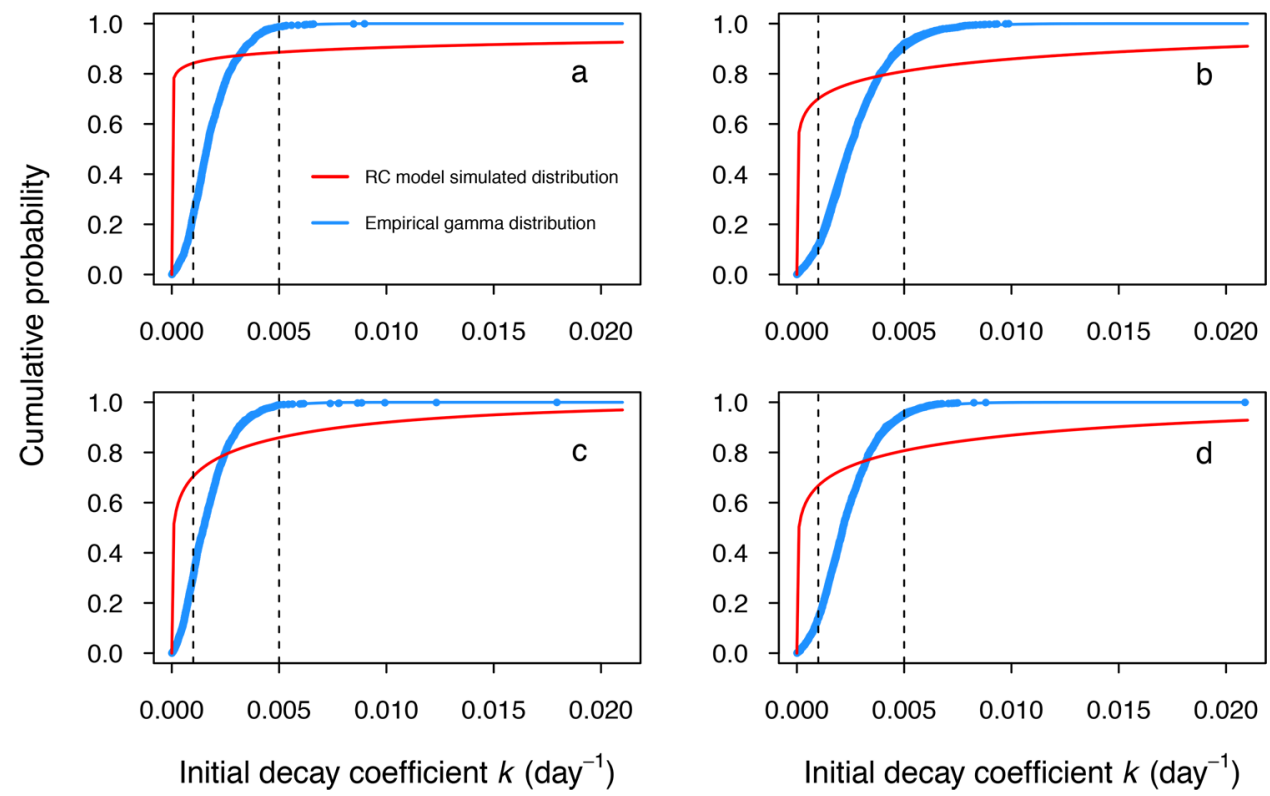

Initial decay coefficient $k\left(\right.$ day $\left.^{-1}\right)$

Figure 2. Probability distribution of initial decay coefficients. RC model-simulated and empirically derived (based on FT-ICR-MS data) distributions are depicted in red and blue, respectively. In the case of the empirical data, the blue dots correspond to apparent decay coefficients $k_{\text {exp }}$ (initial decay coefficient $k$ ), and the blue line shows the fitted distribution. The cumulative distribution function $y=F(k)$, evaluated at $k=x$, is the probability that $k$ takes a value of $x$ or lower. The probability of $k$ to take a value of $x$ or higher is $1-y$. The probability can be converted to percentages of the total (e.g., $0.2=20 \%$ ). In case of the RC model simulation, the "total" refers to the total concentration of DOC, while for the empirical distribution it is the total number of detected molecular formulas with declining intensity. Presented are data for lake Ramsjön nonmanipulated (a) and UVmanipulated (b) treatments, and lake Övre Långsjön nonmanipulated (c) and UV-manipulated (d) treatments. One of the duplicate treatments is shown in each case. Dashed line marks $k=0.001$ and $k=0.005$; accordingly, $k \leq 0.001$ defines the recalcitrant end of the distribution, $0.001<k<$ 0.005 refers to intermediate reactivity, and $k \geq 0.005$ defines the labile end of the distribution. For example, graph (a) shows that the RC model (red) predicts that $84 \%$ of DOC concentration is associated with the $k$ below 0.001 (recalcitrant end), and $11 \%$ is associated with the $k$ above 0.005 (labile end). The empirical distribution (a, blue) suggests that $27 \%$ of molecular formulas are associated with the $k$ below 0.001 (recalcitrant end), and $2 \%$ is associated with the $k$ above 0.005 (labile end).

distributional assumptions were checked using diagnostic plots (SI Figure S6). To better visualize parts of the cumulative gamma distribution we arbitrarily assigned the limits for recalcitrant and labile ends of the distribution at $k=0.001$ and $k=0.005$, respectively. On the basis of these thresholds, we also calculated the proportions of total DOC concentration and 
Table 1. Proportions of the Initial DOC Concentration and Initial Number of Molecular Formulas Associated with a Specific Range of Apparent Decay Coefficients ${ }^{a}$

\begin{tabular}{|c|c|c|c|c|c|c|c|c|}
\hline \multirow[b]{2}{*}{ lake } & \multirow[b]{2}{*}{ treatment } & \multirow[b]{2}{*}{ replicate } & \multicolumn{2}{|c|}{ lower reactivity class (\%) } & \multicolumn{2}{|c|}{$\begin{array}{l}\text { intermediate reactivity class } \\
(\%)\end{array}$} & \multicolumn{2}{|c|}{ higher reactivity class (\%) } \\
\hline & & & DOC & formulas & DOC & formulas & DOC & formulas \\
\hline Ramsjön & unmanipulated & 1 & 84.2 & 26.6 & 4.4 & 71.3 & 11.4 & 2.1 \\
\hline Ramsjön & UV-manipulated & 1 & 70.1 & 14.1 & 10.9 & 75.3 & 19.0 & 10.5 \\
\hline Ramsjön & unmanipulated & 2 & 84.3 & 48.2 & 8.3 & 51.1 & 7.4 & 0.7 \\
\hline Ramsjön & UV-manipulated & 2 & 68.5 & 10.8 & 11.4 & 77.8 & 20.1 & 11.3 \\
\hline Övre Långsjön & unmanipulated & 1 & 70.4 & 33.0 & 15.4 & 65.1 & 14.1 & 1.9 \\
\hline Övre Långsjön & UV-manipulated & 1 & 66.8 & 16.4 & 13.9 & 77.4 & 19.3 & 6.2 \\
\hline Övre Långsjön & unmanipulate & 2 & & & & & & \\
\hline Övre Långsjön & UV-manipulated & 2 & 69.9 & 9.9 & 10.2 & 80.6 & 19.9 & 9.5 \\
\hline
\end{tabular}

${ }^{a}$ Reactivity classes are assigned arbitrarily: $k \leq 0.001$ day $^{-1}$ (lower reactivity class), 0.001 day $^{-1}<k<0.005$ day $^{-1}$ (intermediate reactivity class), and $k \geq 0.005$ day $^{-1}$ (higher reactivity class). Proportions of DOC concentration and proportions of total number of formulas are calculated from the RC model-simulated and empirically derived (based on FT-ICR-MS data) probability distributions of initial decay coefficients, respectively.

total number of formulas associated with higher, intermediate, and lower reactivity sections of the distribution. ${ }^{16}$ Model fitting, calculations, and statistical analyses were performed in $\mathrm{R}$ (version 3.3.0, $\mathrm{R}$ development Core team, 2016).

\section{RESULTS}

Between 1654 and 2001 formulas per water sample were identified with FT-ICR-MS in the different experimental treatments. Between $3.7 \%$ and $10.3 \%$ of the formulas produced exponential trends that were regarded as noise (NRMSE < 35.8, see the Methods section), and the remaining 89.7-96.3\% of the apparent exponential coefficients $k_{\text {exp }}$ were used in the subsequent analysis.

The $k_{\text {exp }}$ acquired from the time series of formula-specific intensities formed a mostly continuous spectrum (Figure 1, and SI Figure S7). On the basis of $k_{\exp }, 85.7 \pm 1.8 \%$ of the rates were classified as net declining, and $13.9 \pm 1.8 \%$ as net increasing.

The range of the empirically derived $k_{\exp }$ was more narrow than the range of initial decay coefficients simulated using RC modeling of bulk DOC decomposition. Specifically, the highest $k$ values predicted by the RC model (concentration-based) were not retrieved from the empirical data (Figure 2 and SI Figure S8).

The cumulative gamma distribution describing the range of empirically derived $k_{\text {exp }}$ suggested that most molecular formulas were associated with intermediate reactivity, while the recalcitrant and labile ends of the distribution were represented by a smaller number of molecular formulas (Figure 2, SI Figure S8, and Table 1). The RC model simulation indicated that recalcitrant compounds constitute the largest share of bulk DOC by concentration, while compounds having intermediate and high reactivity constitute a smaller share (Figure 2, SI Figure S8, and Table 1). Accordingly, the bulk of the DOC did not represent the bulk of individual molecular formulas.

The anticipated effect of pretreatment with UV light on the distribution of reactivities was captured by both the RC modelsimulated and empirically derived cumulative distributions of initial reactivity. Specifically, the proportion of compounds corresponding to the labile end of the distribution increased in both cases (Figure 2, SI Figure S8, and Table 1).

The diversity of empirically derived $k_{\exp }$ declined with increasing intensity of molecular formulas (Figure 3).

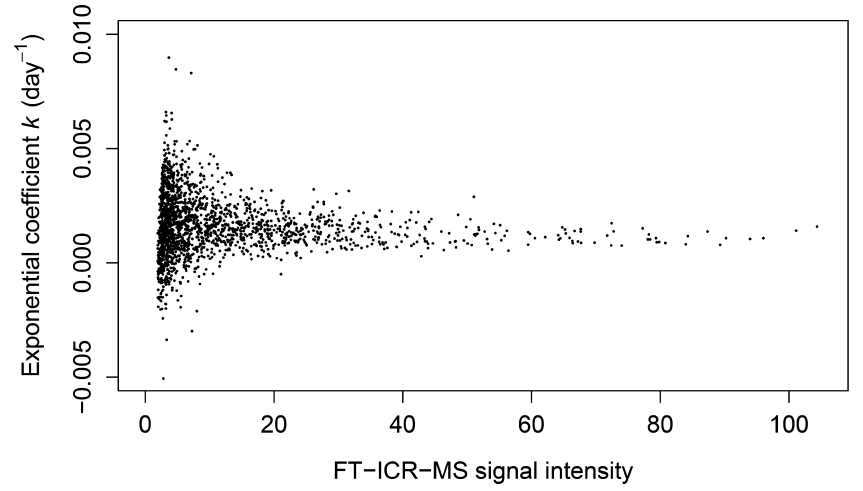

Figure 3. Relationship between the signal intensity (detected at time 0 ) and apparent exponential coefficients of corresponding molecular formulas. Example from lake Ramsjön nonmanipulated treatment (replicate 1). Each dot represents a molecular formula. Positive $k_{\text {exp }}$ (apparent exponential coefficient) correspond to net decay and negative $k_{\exp }$ correspond to net formation/accumulation.

\section{DISCUSSION}

4.1. Continuous Spectrum of DOM Reactivity. Our results support the existence of a reactivity continuum within DOM, consistent with a range of single exponentially decaying components. The picture was compatible for all seven experimental treatments (Figure 1 and SI Figure S7). Thus, we demonstrate a direct link between the kinetics of individual molecular components and the emerging reactivity of complex bulk OM, and present empirical support for the RC model.

Our finding that the DOM constituents align in a reactivity spectrum, rather than aggregate in a few distinct groups of similar decay rates, conceptually speaks against another widespread model approach, i.e., discrete multiexponential decay models.

There were, however, discrepancies in the exact shape of the reactivity distribution between the $\mathrm{RC}$ model simulation and empirically derived data. While these discrepancies do not invalidate the existence of the reactivity continuum that we were able to demonstrate, they highlight the drawbacks of both the FT-ICR-MS and RC model approaches, which we discuss below.

4.2. RC Model Simulated versus Empirical Distribution of Initial Decay Coefficients. We observed a distinct discrepancy between the RC model-simulated and empirically derived probability distributions of initial decay rates. It should 
be taken into account, however, that the RC model simulates what share of DOM compounds by concentration is likely to decompose at a certain rate or faster, while the empirically derived distribution shows what share of the total number of detected formulas is associated with a certain decay rate or higher.

Considering that the probability distribution based on the RC model of bulk DOC and the empirically derived distribution represent different aspects, they should be compared with caution, yet they may also shed light on how DOM concentration is allocated into molecular formulas of different reactivity. The recalcitrant and labile ends of the empirical distribution were represented by a rather small number of molecular formulas, compared to the intermediate section of the distribution, which encompassed the largest portion of the distribution (Figure 2, SI Figure S8, and Table 1). The RC model, in turn, predicted a high concentration of compounds at the recalcitrant end of the spectrum, and a smaller, but still substantial concentration of compounds at the labile end. Hence, despite the fact that the recalcitrant and labile DOM pools are predicted to constitute together up to $90 \%$ of bulk DOM concentration (Figure 2, SI Figure S8, and Table 1), they seem to correspond to a low diversity of molecular formulas. A possible explanation here is that recalcitrant and labile ends of the empirical distribution are underrepresented for various reasons.

4.2.1. Intrinsic Averaging. Multiple previous studies showed that the FT-ICR-MS-detected molecular formula could be clearly related to DOM behavior, e.g., its persistence in lake ${ }^{29}$ and oceanic ${ }^{30}$ water, bio- ${ }^{31-33}$ and photoreactivity, ${ }^{34}$ as well as the sensitivity to thermal alterations. ${ }^{26}$ This suggests that the formula has significant predictive power to describe OM susceptibility to transformation processes. This notion is supported by our own experiment, in which the distribution of apparent decay rates associated with molecular formulas shifted predictably in response to UV pretreatment (Figure 2, SI Figure S8, and Table 1). Yet, the character of empirical distributions suggests a serious limitation of our approach: the range of apparent $k_{\exp }$ retrieved from the mass spectrometry data was narrower than the range of initial $k$-s predicted by the RC model, and the recalcitrant and labile ends of the empirical distribution shrank in favor of the intermediate section of the distribution (Figure 2, SI Figure S8, and Table 1). We believe that the observed effects are primarily caused by the intrinsic averaging of multiple structural isomers behind each formula.

Indeed, each molecular formula identified with FT-ICR-MS encompasses dozens to hundreds of potential isomers. ${ }^{10,35}$ This means that the intensity trends related to each formula actually reflect the integrated behavior of a large group of isomers. Therefore, the apparent exponential decay coefficients that we calculated likely represent the "weighted average" of the reactivity of isomers linked to each molecular formula. It appears that such intrinsic averaging was strong enough to cause the lack of extreme values in the range of apparent $k_{\exp }$ we detect.

Figure 3 indicated low diversity of empirically derived $k_{\text {exp }}$ at higher signal intensities. Possibly, FT-ICR-MS signal intensities may be related to the number of structural isomers. If this is the case, then isomeric averaging may be the reason why peaks with high intensities were associated with low diversity of $k_{\text {exp }}$, similar to the observations of Zark et al. (2017). ${ }^{10}$ Alternatively, intrinsically stable compounds ( $k_{\exp }$ close to zero) would accumulate to relatively high concentrations over time and be possibly represented by large peaks in the mass spectra.

Strictly speaking, we cannot validate the RC model simulation with the empirical mass spectrometry data, or vice versa, due to uncertainties associated with each approach. However, conceptually, we expect the pool of humic lake DOM to be dominated by slowly decaying compounds, as indicated by a small absolute and relative DOC loss in the course of decomposition experiments. ${ }^{16,20}$ Thus, low diversity of molecular formulas on the recalcitrant end of empirical distribution seems inadequate, even if we do not focus on comparison with the RC model-simulated distribution. Having an indication that FT-ICR-MS was unable to retrieve the full spectrum of DOM reactivity, we argue that the discrepancy between RC model-simulated and empirical data is mainly caused by analytical constraints, rather than by flaws in the reactivity continuum theory. Moreover, since there was a wide range of decay (and formation) rates across the detected molecular formulas, the variability in reactivity within molecular formulas did not completely obscure the formula-specific kinetics. Accordingly, we were able to retrieve a continuum of reactivity and capture the effects of pretreatment with UV light. This justifies our approach to view individual formula as a reactive type.

Our results, demonstrating patterns of reactivity across molecular formulas and the discrepancy of these patterns from the conceptual expectations, shed light on the isomeric complexity of individual molecular formulas in the DOM mixture. This is an important outcome that warrants further research.

4.2.2. Overlap of Loss and Formation Rates. By its nature, the bulk DOM decomposition data reflects what fraction of DOM was completely mineralized to $\mathrm{CO}_{2}$. However, decomposition of specific compounds often includes several steps and intermediate products, e.g., in cases where molecules are degraded into monomers by extracellular enzymes. ${ }^{36}$ The intermediate products decompose further, but also simultaneously build up from the preceding reaction, leading to an overall nontrivial decay behavior. If, for a given formula, formation and loss rates are similar in magnitude, then the degradation will not be apparent in the FT-ICR-MS-derived data. If decay exceeds formation, then the intensity will show an apparent decline, and vice versa. Such overlap of loss and formation rates can obscure the variable dynamics of molecules and prevent us from getting a detailed picture of kinetic processes underlying the $\mathrm{OM}$ behavior.

The RC model implicitly considers overlaps in the formation and decay rates when parametrizing the bulk DOM mass loss curve. This is reflected in early definitions, e.g., by Boudreau and Ruddick (1991): "The apparent reactivity of a continuum mixture of organic matter types is a function of the changing composition of the mass with time". ${ }^{6}$ The bulk DOM mass loss curve, and hence the $\mathrm{RC}$ model parameter estimates, are strongly influenced by the initial amount of highly reactive material. These highly reactive DOM components which decay fast are least influenced by simultaneous formation. Since these components are strongly responsible for the shape of decay curve and the RC model parameters, one can infer that the RC model outcome should not be seriously confounded by simultaneous loss and formation. We suggest that to a certain extent these considerations would also hold for the FT-ICR-MS data, i.e., in the FT-ICR-MS data, the formula-specific decay and formation rates are also integrated into apparent decay 
coefficients. In the same way as described for the RC model, the most reactive components should be least confounded by simultaneous formation.

4.2.3. Accumulating Compounds. One of the consequences of simultaneous loss and formation is the apparent presence of accumulating compounds. Around 14\% of molecular formulas displayed negative $k_{\text {exp }}$, indicating net increasing concentration. The observed increase of some compounds highlights the fact that the spectrum of DOM reactivity in reality integrates both degrading and accumulating compounds. This is consistent with previous observations that heterotrophic bacteria in parallel with degradation of DOM also produce new compounds, ${ }^{37}$ including humic-like and recalcitrant DOM. $^{38-40}$ Since mass loss dominates bulk DOM decomposition, accumulation of compounds is implicitly accounted for by the RC model; if present, accumulation would simply lower an apparent decay rate. However, to describe the empirical distribution (Figure 2 and SI Figure S8), we had to eliminate formulas with net increasing signal, because the gamma distribution only allows $k_{\exp }$ to be positive. This should have shifted our empirical distribution toward a higher reactivity.

Since the mass spectrometry data cannot account for absolute concentration of compounds we could not evaluate how much the accumulating compounds contributed to the bulk DOM pool. In other words, we cannot evaluate by how much the RC model overestimated the pool of slowly decaying $\mathrm{DOM}$ due to the presence of accumulating compounds. However, in the context of bulk DOM, the concentration of potentially accumulating decomposition products (assessed as carbon concentration) cannot exceed the initial concentration of the source DOM. Similarly, only a fraction of bulk organic carbon becomes assimilated by heterotrophic microbes during decomposition and can be further converted into accumulating metabolites. For example, Guillemette et al. ${ }^{37}$ estimated the production rate of roughly $0.1 \%$ fluorescent DOM per day by lake bacterioplankton. These considerations justify that the RC model does not specifically distinguish accumulating compounds. In certain cases OM decomposition is characterized by an initial lag phase and other models, e.g., the Weibull RC model, may be considered as an alternative. ${ }^{18}$ While, theoretically, an initial lag phase might be caused by relatively high rates of simultaneous DOM production, it can also be explained by nutrient limitation and other decompositioninhibiting processes. ${ }^{41}$ Regardless, we did not observe an initial lag-phase in the bulk DOM decay trend in our experiment (Figure 1).

4.2.4. Additional Considerations. On the basis of the preceding discussion we propose that the intrinsic averaging is a primary concern that limited interpretation of our data and reconciliation of RC model and FT-ICR-MS approaches. Some additional considerations, however, must be mentioned as well.

A previous study indicates that some fast-degrading compounds, such as saccharides, are likely to be insufficiently ionized in negative ESI mode and therefore remain outside of the FT-ICR-MS analytical window. ${ }^{42}$ The difficulty to retrieve high apparent decay coefficients from the mass spectrometry data would be an expected outcome of such selectivity and would contribute to the absence of high $k_{\exp }$ in our data. Moreover, since we could only obtain apparent decay coefficients for formulas that were present through the whole experiment, formulas that disappeared within hours and days were not considered in our empirical data.
In the discussion of the shortcomings associated with our analytical approach it should be stressed that FT-ICR-MS resolves molecular information from complex mixtures like DOM in far more detail than any other analytical method available to date. Therefore, despite the limitations, FT-ICRMS offered the best available means to address the objectives of our study.

The limitations of the RC model based on DOC data are described in detail in Koehler and Tranvik (2015). Briefly, in certain cases, the data suggest violation of the RC model assumption. Yet, such cases often result from limitations in the data (too few data points, the observation time is not sufficient) rather than from conceptual flaws of the RC theory. ${ }^{18}$ Possible experimental design solutions as well as aspects of how to compare RC model performance with that of other models are described elsewhere. ${ }^{16,18,20}$

4.3. Treatment Effects. Both the RC model and the empirical data captured the anticipated effect of DOM pretreatment with the UV light: in both cases the probability distribution shifted toward an increase in the labile pool (Figure 2 and SI Figure S8). Hence, despite the fact that the decay of labile peaks is underrepresented in the mass spectrometry data, the technique still adequately reflects the shifts in DOM behavior due to changes in composition. It has previously been shown that a range of highly labile low molecular weight compounds are produced upon solar exposure of DOM, typically with molecular weights of about 200 or less. ${ }^{43,44}$ These compounds would be expected to largely escape the analytical window of FT-ICR-MS, and also be mineralized too rapidly to be detected at the time scale and temporal sampling resolution of our experiment. ${ }^{44}$ Hence, both the RC model and the empirical distributions demonstrated here suggest that the UVstimulation of OM decay includes not only highly labile compounds such as low molecular weight carboxylic acids, but also larger and more slowly decomposing photoproduced molecules.

4.4. Implications. While the RC model was indirectly tested before for its performance to describe decomposition in different systems/data sets and using mathematical indicators, ${ }^{15,16,18,20}$ we here present a direct empirical support for the central idea behind the theory of reactivity continuum. Our results clearly demonstrate the existence of the continuum of reactivity within bulk $\mathrm{OM}$, as evidenced by a spectrum of molecular formula-specific apparent exponential decay coefficients. This spectrum was detectable despite the effect of isomeric averaging, which likely averaged-out extreme decay rates and made each molecular mass appear to decay at a more average, intermediate value. Methods for our in-depth approach became available only recently, with the advent of ultrahighresolution mass spectrometry. Our work contributes to exploration of its potential to study the kinetics of OM decay.

\section{ASSOCIATED CONTENT}

\section{Supporting Information}

The Supporting Information is available free of charge on the ACS Publications website at DOI: 10.1021/acs.est.7b02876.

Eight figures (S1-S8) and one table (Table S1). Figures S1 and S2 are accompanied by the explanation of the methods used to derive the figures (PDF). 


\section{AUTHOR INFORMATION}

\section{Corresponding Author}

*Phone: +46184712707; e-mail: alina.mostovaya@ebc.uu.se (A.M.).

\section{ORCID}

Alina Mostovaya: 0000-0002-5884-1684

\section{Notes}

The authors declare no competing financial interest.

\section{ACKNOWLEDGMENTS}

We thank Karólína Einarsdóttir and Jan Johansson for assistance with the experiment; Katrin Klaproth and Helena Osterholz for assistance with the processing of mass spectrometry data; Moritz Buck and Yevgen Ryeznik for suggestions regarding the $\mathrm{R}$ code. Exemplary $\mathrm{R}$ code for analysis of decomposition data using the gamma reactivity continuum model is available on ResearchGate (DOI: 10.13140/RG.2.1.4471.3684). Other data related to this study is available upon request from A. Mostovaya (alina.mostovaya@ebc.uu.se). The study was financed by the Swedish Research Council (grant 2011-3475-88773-67), by the Nordforsk (DOMQUA project; 60501), and by the Knut and Alice Wallenberg Foundation (grant KAW 2013.0091). B.K. acknowledges funding by the Ingmar Bergman Foundation 2016.

\section{REFERENCES}

(1) Benner, R.; Fogel, M. L.; Sprague, E. K.; Hodson, R. E. Depletion of $13 \mathrm{C}$ in lignin and its implication for stable carbon isotope studies. Nature 1987, 329 (6141), 708-710.

(2) Westrich, J. T.; Berner, R. A. The role of sedimentary organic matter in bacterial sulfate reduction: The G model tested. Limnol. Oceanogr. 1984, 29 (2), 236-249.

(3) Amon, R. M. W.; Benner, R. Bacterial utilization of different size classes of dissolved organic matter. Limnol. Oceanogr. 1996, 41 (1), $41-51$.

(4) Guillemette, F.; del Giorgio, P. A. Reconstructing the various facets of dissolved organic carbon bioavailability in freshwater ecosystems. Limnol. Oceanogr. 2011, 56 (2), 734-748.

(5) Carpenter, S. R. Decay of heterogenous detritus: a general model. J. Theor. Biol. 1981, 89 (4), 539-547.

(6) Boudreau, B. P.; Ruddick, B. R. On a reactive continuum representation of organic matter diagenesis. Am. J. Sci. 1991, 291 (5), 507-538.

(7) Bosatta, E.; Ågren, G. I. The power and reactive continuum models as particular cases of the q-theory of organic matter dynamics. Geochim. Cosmochim. Acta 1995, 59 (18), 3833-3835.

(8) Minor, E. C.; Steinbring, C. J.; Longnecker, K.; Kujawinski, E. B. Characterization of dissolved organic matter in Lake Superior and its watershed using ultrahigh resolution mass spectrometry. Org. Geochem. 2012, 43, 1-11.

(9) Kellerman, A. M.; Dittmar, T.; Kothawala, D. N.; Tranvik, L. J. Chemodiversity of dissolved organic matter in lakes driven by climate and hydrology. Nat. Commun. 2014, 5, 1-8.

(10) Zark, M.; Christoffers, J.; Dittmar, T. Molecular properties of deep-sea dissolved organic matter are predictable by the central limit theorem: Evidence from tandem FT-ICR-MS. Mar. Chem. 2017, 191, 9-15.

(11) Riedel, T.; Dittmar, T. A method detection limit for the analysis of natural organic matter via Fourier transform ion cyclotron resonance mass spectrometry. Anal. Chem. 2014, 86, 8376-8382.

(12) Aumont, O.; van Hulten, M.; Roy-Barman, M.; Dutay, J.-C.; Éthé, C.; Gehlen, M. Variable reactivity of particulate organic matter in a global ocean biogeochemical model. Biogeosciences 2017, 14, 23212341.
(13) Sierra, C. A.; Harmon, M. E.; Perakis, S. S. Decomposition of heterogeneous organic matter and its long-term stabilization in soils. Ecol. Monogr. 2011, 81 (4), 619-634.

(14) Manzoni, S.; Piñeiro, G.; Jackson, R. B.; Jobbágy, E. G.; Kim, J. H.; Porporato, A. Analytical models of soil and litter decomposition: solutions for mass loss and time-dependent decay rates. Soil Biol. Biochem. 2012, 50, 66-76.

(15) Vähätalo, A. V.; Aarnos, H.; Mäntyniemi, S. Biodegradability continuum and biodegradation kinetics of natural organic matter described by the beta distribution. Biogeochemistry 2010, 100 (1-3), 227-240.

(16) Koehler, B.; Von Wachenfeldt, E.; Kothawala, D.; Tranvik, L. J. Reactivity continuum of dissolved organic carbon decomposition in lake water. J. Geophys. Res. Biogeosci. 2012, 117 (1), 1-15.

(17) Guillemette, F.; McCallister, S. L.; del Giorgio, P. A. Differentiating the degradation dynamics of algal and terrestrial carbon within complex natural dissolved organic carbon in temperate lakes. J. Geophys. Res.: Biogeosci. 2013, 118 (3), 963-973.

(18) Koehler, B.; Tranvik, L. J. Reactivity continuum modeling of leaf, root, and wood decomposition across biomes. J. Geophys. Res.: Biogeosci. 2015, 120 (7), 1196-1214.

(19) Forney, D. C.; Rothman, D. H. Common structure in the heterogeneity of plant-matter decay. J. R. Soc., Interface 2012, 9 (74), $2255-2267$.

(20) Mostovaya, A.; Koehler, B.; Guillemette, F.; Brunberg, A.-K.; Tranvik, L. J. Effects of compositional changes on reactivity continuum and decomposition kinetics of lake dissolved organic matter. J. Geophys. Res.: Biogeosci. 2016, 121 (7), 1733-1746.

(21) Xian, F.; Hendrickson, C. L.; Marshall, A. G. High resolution mass spectrometry. Anal. Chem. 2012, 84 (2), 708-719.

(22) Dittmar, T.; Stubbins, A. Dissolved organic matter in aquatic systems. In Treatise on Geochemistry, $2^{\text {nd }}$ ed.; Holland, H. D., Turekian, K. K., Eds.; Elsevier: Oxford, 2014; pp 125-156.

(23) Hawkes, J. A.; Dittmar, T.; Patriarca, C.; Tranvik, L. J.; Bergquist, J. Evaluation of the Orbitrap mass spectrometer for the molecular fingerprinting analysis of natural dissolved organic matter. Anal. Chem. 2016, 88, 7698-7704.

(24) Seidel, M.; Yager, P. L.; Ward, N. D.; Carpenter, E. J.; Gomes, H. R.; Krusche, A. V.; Richey, J. E.; Dittmar, T.; Medeiros, P. M. Molecular-level changes of dissolved organic matter along the Amazon River-to-ocean continuum. Mar. Chem. 2015, 177, 218-231.

(25) Green, N. W.; Perdue, E. M.; Aiken, G. R.; Butler, K. D.; Chen, H.; Dittmar, T.; Niggemann, J.; Stubbins, A. An intercomparison of three methods for the large-scale isolation of oceanic dissolved organic matter. Mar. Chem. 2014, 161, 14-19.

(26) Hawkes, J. A.; Hansen, C. T.; Goldhammer, T.; Bach, W.; Dittmar, T. Molecular alteration of marine dissolved organic matter under experimental hydrothermal conditions. Geochim. Cosmochim. Acta 2016, 175, 68-85.

(27) Boudreau, B. P.; Arnosti, C.; Jørgensen, B. B.; Canfield, D. E. Comment on "Physical model for the decay and preservation of marine organic carbon. Science 2008, 319, 1616b.

(28) Olson, J. S. Energy storage and the balance of producers and decomposers in ecological systems. Ecology 1963, 44 (2), 322-331.

(29) Kellerman, A. M.; Kothawala, D. N.; Dittmar, T.; Tranvik, L. J. Persistence of dissolved organic matter in lakes related to its molecular characteristics. Nat. Geosci. 2015, 8 (6), 454-457.

(30) Lechtenfeld, O. J.; Kattner, G.; Flerus, R.; McCallister, S. L.; Schmitt-Kopplin, P.; Koch, B. P. Molecular transformation and degradation of refractory dissolved organic matter in the Atlantic and Southern Ocean. Geochim. Cosmochim. Acta 2014, 126, 321-337.

(31) Riedel, T.; Zark, M.; Vähätalo, A. V.; Niggemann, J.; Spencer, R. G. M.; Hernes, P. J.; Dittmar, T. Molecular signatures of biogeochemical transformations in dissolved organic matter from ten world rivers. Front. Earth Sci. 2016, 4, Article No. 85.

(32) Sleighter, R. L.; Cory, R. M.; Kaplan, L. A.; Abdulla, H. A. N.; Hatcher, P. G. A coupled geochemical and biogeochemical approach to characterize the bioreactivity of dissolved organic matter from a 
headwater stream. J. Geophys. Res.: Biogeosci. 2014, 119 (8), 15201537.

(33) D’Andrilli, J. D.; Cooper, W. T.; Foreman, C. M.; Marshall, A. G. An ultrahigh-resolution mass spectrometry index to estimate natural organic matter lability. Rapid Commun. Mass Spectrom. 2015, 29, 2385-2401.

(34) Stubbins, A.; Spencer, R. G. M.; Chen, H.; Hatcher, P. G.; Mopper, K.; Hernes, P. J.; Mwamba, V. L.; Mangangu, A. M.; Wabakanghanzi, J. N.; Six, J. Illuminated darkness: molecular signatures of Congo River dissolved organic matter and its photochemical alteration as revealed by ultrahigh precision mass spectrometry. Limnol. Oceanogr. 2010, 55 (4), 1467-1477.

(35) Hertkorn, N.; Ruecker, C.; Meringer, M.; Gugisch, R.; Frommberger, M.; Perdue, E. M.; Witt, M.; Schmitt-Kopplin, P. High-precision frequency measurements: Indispensable tools at the core of the molecular-level analysis of complex systems. Anal. Bioanal. Chem. 2007, 389 (5), 1311-1327.

(36) Arnosti, C. Microbial extracellular enzymes and the marine carbon cycle. Annu. Rev. Mar. Sci. 2011, 3, 401-425.

(37) Guillemette, F.; del Giorgio, P. A. Simultaneous consumption and production of fluorescent dissolved organic matter by lake bacterioplankton. Environ. Microbiol. 2012, 14 (6), 1432-1443.

(38) Brophy, J. E.; Carlson, D. J. Production of biologically refractory dissolved organic carbon by natural seawater microbial populations. Deep-Sea Res., Part A 1989, 36 (4), 497-507.

(39) Tranvik, L. J. Microbial transformation of labile dissolved organic matter into humic-like matter in seawater. FEMS Microbiol. Ecol. 1993, 12 (3), 177-183.

(40) Ogawa, H.; Amagai, Y.; Koike, I.; Kaiser, K.; Benner, R. Production of refractory dissolved organic matter by bacteria. Science 2001, 292 (5518), 917-920.

(41) Cornwell, W. K.; Weedon, J. T. Decomposition trajectories of diverse litter types: a model selection analysis. Methods Ecol. Evol. 2014, 5 (2), 173-182.

(42) Raeke, J.; Lechtenfeld, O. J.; Wagner, M.; Herzsprung, P.; Reemtsma, T. Selectivity of solid phase extraction of freshwater dissolved organic matter and its effect on ultrahigh resolution mass spectra. Environ. Sci. Process. Impacts 2016, 18 (7), 918-927.

(43) Moran, M. A.; Zepp, R. G. Role of photoreactions in the formation of biologically labile compounds from dissolved organic matter. Limnol. Oceanogr. 1997, 42 (6), 1307-1316.

(44) Bertilsson, S.; Tranvik, L. J. Photochemical transformation of dissolved organic matter in lakes. Limnol. Oceanogr. 2000, 45 (4), 753-762. 